\title{
Way to go, RNA
}

\section{JOEL G. BELASCO}

Kimmel Center for Biology and Medicine at the Skirball Institute and Department of Microbiology, New York University School of Medicine, New York, New York 10016, USA

Although the field of RNA biology can trace its origin to the early 20th century, it first achieved prominence in the decade from 1955 to 1965 with pivotal breakthroughs that included the discovery of ribosomes, tRNA, and mRNA and the cracking of the genetic code. The next 30 years witnessed many other seminal discoveries, such as mRNA splicing, catalytic RNA, and regulatory RNA. To meet the needs of this flourishing field of investigation, those four decades concluded 20 years ago with the founding of the RNA Society and its monthly journal RNA.

Progress in the field since then is well illustrated by advances in any number of subdisciplines. Consider the two principal fates of mRNA: translation and degradation. By 1990, much was understood about translation in bacterial and eukaryotic cells, despite the dearth of structural detail at that time. In contrast, descriptions of mRNA degradation were still largely phenomenological, even in so well studied an organism as Escherichia coli. By then, it had been established that the lifetimes of bacterial mRNAs, and even of distinct segments within the same transcript, can differ significantly from one another and that such differences in longevity can have a profound effect on gene expression. However, the basis for these differences remained a mystery, as emerging evidence that bacterial mRNA lifetimes might be governed by as yet uncharacterized features of the $5^{\prime}$ untranslated region was difficult to reconcile with the apparent absence of $5^{\prime}$ exoribonuclease activity in E. coli, whose assortment of ribonucleases is limited to endonucleases and $3^{\prime}$ exonucleases. Because those $3^{\prime}$ exonucleases were known to be impeded upon encountering base-paired regions, such as the stem-loop structures typically present at the $3^{\prime}$ end of bacterial mRNAs, it was hypothesized that RNA degradation in bacterial cells generally begins with internal cleavage by a low-specificity endonuclease to generate a $5^{\prime}$ fragment susceptible to attack by $3^{\prime}$ exonucleases, such as polynucleotide phosphorylase (PNPase) and RNase II, and a $3^{\prime}$ fragment presumed to undergo further endonucleolytic fragmentation followed by exonucleolytic digestion. However, this rudimentary mechanism provided no clue as to the identity of the endonuclease, nor did it ex-

\footnotetext{
Corresponding author: joel.belasco@med.nyu.edu

Article and publication date are at http://www.rnajournal.org/cgi/doi/ 10.1261/rna.050773.115. Freely available online through the RNA Open Access option.
}

plain how base-paired regions ever get degraded or why the $3^{\prime}$ product of the initial endonucleolytic event is typically shorter lived (i.e., more susceptible to cleavage) than its intact precursor.

The early 1990s witnessed some key discoveries, including the identification of RNase E, which targets single-stranded regions, as the endonuclease most important for mRNA degradation in E. coli and the observation that it forms a multisubunit complex (the RNA degradosome) with PNPase and other proteins. Concurrent studies showed that base-pairing at the $5^{\prime}$ terminus can prolong the lifetime of RNA in E. coli by impeding internal cleavage by RNase $\mathrm{E}$, a counterintuitive finding that corroborated the influence of the $5^{\prime}$ untranslated region on transcript stability without suggesting a mechanism. In addition, poly(A) polymerase, an enzyme activity long known to exist in bacteria but largely ignored, perhaps because of the ephemeral nature of bacterial poly(A) tails, was found to destabilize certain RNA decay intermediates by an unknown mechanism.

This was roughly the state of knowledge about bacterial mRNA degradation when the first issue of RNA was published 20 years ago-a lot of phenomenology but little real understanding. What followed was a profusion of mechanistic insights, beginning with revelations about degradative events at the $3^{\prime}$ end. Among the first was the discovery that poly(A)polymerase facilitates the degradation of decay intermediates that end with a stem-loop by adding a $3^{\prime}$-terminal single-stranded tail that PNPase and the RNA helicase RhlB (another degradosome component) can use as a beachhead for launching an assault on the stem-loop preceding it. At last, a mechanism for degrading base-paired regions of RNA was in hand. Interestingly, due to the reversibility of RNA phosphorolysis, PNPase was found not only to degrade RNA in bacteria but also to add heteropolymeric tails that are A-rich, enabling this enzyme to single-handedly create unstructured $3^{\prime}$ ends on which it can reverse course and launch an exonucleolytic attack. In contrast, the hydrolytic $3^{\prime}$ exonuclease RNase II, which does not associate with an E. coli helicase, proved unexpectedly to have a protective rather than a destructive effect on base-paired $3^{\prime}$ ends due to its

(C) 2015 Belasco This article, published in $R N A$, is available under a Creative Commons License (Attribution-NonCommercial 4.0 International), as described at http://creativecommons.org/licenses/by-nc/4.0/. 
propensity to selectively remove poly(A) tails without damaging the $3^{\prime}$ stem-loop. Furthermore, the discovery of tmRNAmediated trans-translation explained what happens when $3^{\prime}$ exonucleases advancing toward the $5^{\prime}$ end encounter translating ribosomes moving in the opposite direction. By releasing ribosomes trapped at the $3^{\prime}$ end of mRNA decay intermediates that lack a stop codon, tmRNA was found to unmask those ends and render them susceptible to $3^{\prime}$-exonucleolytic attack.

Important progress toward understanding the role of $5^{\prime}$ ends in bacterial mRNA degradation soon followed. A key insight came from the surprising discovery in 1998 that the rate at which RNase E cleaves RNA at internal sites is greatly influenced by the number of phosphates at the $5^{\prime}$ end, monophosphorylated substrates being cut as much as 10-100 times faster than their triphosphorylated equivalents. This unprecedented property, which was later shown to be a consequence of a discrete $5^{\prime}$-monophosphate-binding pocket on the surface of this endonuclease, helped to explain the very short lifetime of most $3^{\prime}$-terminal decay intermediates, which generally bear a single phosphate at the $5^{\prime}$ end. However, this property alone was insufficient to account for the influence of $5^{\prime}$-terminal base-pairing on the longevity of primary transcripts, whose triphosphorylated $5^{\prime}$ ends cannot interact with RNase E. The explanation for the latter phenomenon required the subsequent discovery of the RNA pyrophosphohydrolase RppH, which converts the triphosphorylated 5' ends of primary transcripts to monophosphorylated termini that can interact productively with RNase E if single-stranded, thereby triggering the degradation of many RNAs by a $5^{\prime}$ end-dependent mechanism.

Meanwhile, the advent of whole genome sequencing in the late 1990s revealed major disparities in the ribonucleolytic arsenals present in different bacterial species, making it clear that $E$. coli could not be considered a universal paradigm for bacterial mRNA degradation. For example, despite its critical role in E. coli, where it is essential for cell growth, RNase E proved to be absent from many other bacterial species, including Bacillus subtilis and a number of important human pathogens. Instead, those organisms were found to contain alternative ribonucleases not present in E. coli, such as RNase Y (a low-specificity endonuclease) and RNase J. The subsequent discovery that RNase $\mathrm{J}$ is a $5^{\prime}$-monophosphatedependent $5^{\prime}$ exonuclease explained the rapid degradation of monophosphorylated decay intermediates in species that lack RNase E as well as the polar influence of stalled ribosomes, which had previously been shown to selectively stabi- lize the RNA segment downstream from the stall site in $B$. subtilis.

In recent years, considerable progress has also been made toward understanding the influence of ancillary factors on bacterial mRNA degradation, most notably the effect of noncoding RNAs that base-pair with mRNA. Though first discovered in the 1980s, the widespread influence of partially complementary small RNAs (sRNAs) and fully complementary antisense RNAs was not appreciated until many more of them were identified, beginning in the mid-1990s. It soon became clear that noncoding RNAs can either down-regulate or up-regulate gene expression, frequently by controlling the accessibility of ribosome binding sites in the mRNAs to which they bind but also by altering rates of mRNA decay. Often the influence on mRNA decay is a secondary consequence of the effect on translation, due to diminished or increased protection of the mRNA by ribosomes. However, sometimes it appears to be a direct effect of the ability of a bound regulatory RNA to create or mask an internal cleavage site or to recruit RNase E, either because the sRA is monophosphorylated or because it is associated with Hfq, an Smlike protein chaperone that can bind to RNase E.

In the meantime, parallel studies of mRNA decay in eukaryotic cells have identified a variety of degradative pathways triggered by poly(A) tail removal, decapping, or endonucleolytic cleavage. Some of these eukaryotic pathways and the enzymes on which they rely have counterparts in bacteria, suggesting the divergent evolution of ancient RNA degradation mechanisms that date back to a common progenitor.

As much as has been learned about mRNA degradation in the last 20 years, even more is not yet understood, and the next 20 years of progress in elucidating the mechanisms and biological impact of this critical regulatory process in each of the three domains of life promise to be fascinating. More generally, what do the coming decades have in store for the field of RNA biology as a whole? The essence of hypothesis-driven scientific investigation is to resolve known unknowns, which by their nature are definable if countless. As for unknown unknowns, there surely are many of those as well, RNA biology having repeatedly been reinvigorated by unanticipated discoveries that have enabled the field to reinvent itself.

Find a seat. The sequel is about to begin.

\section{Acknowledgments}

This work is supported by NIH grant R01GM035769. 

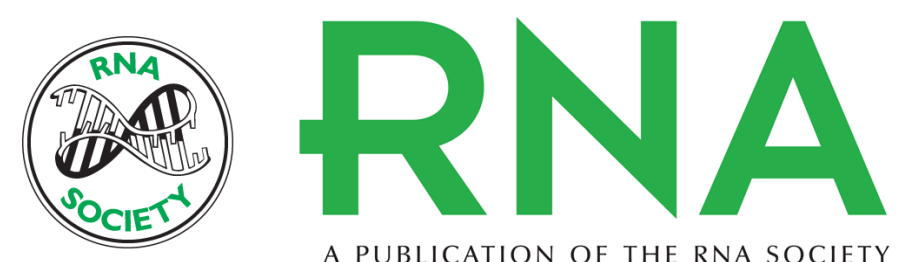

A PUBLICATION OF THE RNA SOCIETY

\section{Way to go, RNA}

Joel G. Belasco

RNA 2015 21: 565-566

Open Access Freely available online through the RNA Open Access option.

Creative This article, published in RNA, is available under a Creative Commons License

Commons (Attribution-NonCommercial 4.0 International), as described at

License http://creativecommons.org/licenses/by-nc/4.0/.

Email Alerting Receive free email alerts when new articles cite this article - sign up in the box at the Service top right corner of the article or click here. 